\title{
Peristaltic Sisko nano fluid in an asymmetric channel
}

\author{
Noreen Sher Akbar
}

Received: 21 January 2013/Accepted: 15 February 2013/Published online: 22 June 2013

(C) The Author(s) 2013. This article is published with open access at Springerlink.com

\begin{abstract}
The present article deals with the peristaltic flow of a Sisko nanofluid in an asymmetric channel. Here the governing flow equations for nano Sisko fluid are formulated in Cartesian coordinates system. The flow analysis is developed in a wave frame of reference moving with velocity of the wave $c$. The fourth- and fifth-order RungeKutta-Fehlberg method is used to calculate the numerical solutions of simplified coupled nonlinear equations and find the expressions of velocity, stream function, pressure rise, temperature and nanoparticle phenomena graphically. Numerical solutions are also presented in tabulated form.
\end{abstract}

Keywords Peristaltic flow · Nano fluid · Sisko fluid model $\cdot$ Asymmetric channel $\cdot$ Numerical solution

\section{Introduction}

Peristalsis is the wavelike strapping contractions in tubular structures, particularly organs of the digestive system such as the oesophagus and the bowels. Peristalsis is characterized by swap reduction and leisure, which pushes foodstuff in the course of the digestive area towards its let go at the anus. Worms impel themselves through peristaltic association. Latham (Latham 1966) first discussed the peristaltic flow in his MS thesis in 1960. According to him a peristaltic force is an organic siphon which employs episodic wavelike squeezing movement which voyages down a vessel and forces the stuffing of the vessel. After Latham, peristaltic pumping feature in detail was offered by Jaffrin and Shapiro

N. S. Akbar ( $\square)$

DBS\&H, CEME, National University of Sciences

and Technology, Islamabad, Pakistan

e-mail: noreensher@yahoo.com
(1971). Elshehawey et al. (2000) presented peristaltic motion of a generalized Newtonian fluid under the effect of transverse magnetic field. Peristaltic pumping by a sinusoidal travelling wave in the porous walls of a two-dimensional channel filled with a viscous incompressible conducting fluid under the effect of transverse magnetic field is investigated theoretically and graphically by El-Shehawey et al. (2002) Peristaltic transport of an incompressible viscous fluid in an inclined asymmetric channel through a porous medium is studied by Kothandapani and Srinivas (2008) under longwavelength and low Reynolds number assumptions. In another article, Srinivas and Kothandapani (2009) discussed the effects of heat and mass transfer on peristaltic transport in a porous space with compliant walls. They considered that the fluid is electrically conducting in the presence of a uniform magnetic field. Analytic solution is carried out under long-wavelength and low Reynolds number approximations. A number of systematic, geometric and tentative (Mekheimer 2008; Akbar et al. 2012a; Akbar and Nadeem 2012a Nadeem and Akbar 2010) studies of peristaltic flows of different fluids have been reported under different circumstances with orientation to physiological and mechanical situations.

Nanofluids simultaneously make and disperse the nanoparticles directly into a base fluid and prevent oxidation of pure metal particles. The nanofluid has been highly developed after the first investigation done by Choi (1995) who showed substantial growth of heat related in suspensions of copper or aluminium nanoparticles in water and other liquids. Heat transfer enhancement using nanofluids in forced convection flows is discussed by Marga et al. (2005) They depicted that enclosure of nanoparticles into the underneath fluids has bent a considerable expansion of the heat transfer coefficient that evidently increases with an increase of the particle absorption. Sadik and 
Pramuanjaroenkij (2009) give the review of convective heat transfer enhancement with nanofluids. Natural convection heat transfer in a nanofluid-filled trapezoidal enclosure is investigated by Saleh et al. (2011). They developed a new correlation for the average Nusselt number as a function of the angle of the sloping wall, effective thermal conductivity and viscosity as well as Grashof number. Another chronological study on nanofluids is cited in the Refs. (Akbar and Nadeem 2011, 2012b; Aziz and Khan 2012; Hamad and Ferdows 2012; Akbar et al. 2012b, c; Akram et al. 2012).

The aim of the current study was to look at the peristaltic flow of a nano Sisko fluid in an asymmetric channel. the nano Sisko model for peristaltic flow problems. So consequently, to fill this stare in the current psychoanalysis we have discussed the peristaltic flow of Sisko fluid model with nano particle phenomena in an asymmetric channel. Next section describes the mathematical formulation of the problem. Numerical solution graphically for velocity, pressure rise, temperature, nanoparticle phenomena and streamlines are presented in section three. The summary of the present work is presented in the last section of the article.

\section{Mathematical formulation}

Let us consider an incompressible Sisko fluid with nano particle in an asymmetric channel of width $d_{1}+d_{2}$. The channel has a sinusoidal wave propagating with constant speed $c$ on the channel walls induces the flow. The asymmetry of the channel is due to different amplitudes. Temperature $\bar{T}_{0}, \bar{T}_{1}$ and Nano particle concentrations $\bar{C}_{0}, \bar{C}_{1}$ are given for the upper and lower wall of the channel. The wall surfaces are selected that satisfy the following expressions:

$$
\begin{aligned}
& Y=H_{1}=d_{1}+a_{1} \cos \left[\frac{2 \pi}{\lambda}\left(X-c_{1} t\right)\right], \\
& Y=H_{2}=-d_{2}-b_{1} \cos \left[\frac{2 \pi}{\lambda}\left(X-c_{1} t\right)+\phi\right] .
\end{aligned}
$$

In the above equations $a_{1}$ and $b_{1}$ are the waves amplitudes, $\lambda$ is the wave length, $d_{1}+d_{2}$ is the channel width, $c_{1}$ is the wave speed, $t$ is the time, $X$ is the direction of wave propagation and $Y$ is perpendicular to $X$. The phase difference $\phi$ varies in the range $0 \leq \phi \leq \pi$. When $\phi=0$ then symmetric channel with waves out of phase can be described and for $\phi=\pi$ the waves are in phase. Moreover, $a_{1}, b_{1}, d_{1}, d_{2}$ and $\phi$ satisfy the following relation:

$a_{1}^{2}+b_{1}^{2}+2 a_{1} b_{1} \cos \phi \leq\left(d_{1}+d_{2}\right)^{2}$.

The coordinates, velocity components and pressure between fixed and wave frames are related by the following transformations:

$$
\begin{aligned}
\bar{x} & =\bar{X}-c_{1} t, \quad \bar{y}=\bar{Y}, \bar{u}=\bar{U}-c_{1}, \bar{v}=\bar{V}, p(\bar{x}) \\
& =P(\bar{X}, t),
\end{aligned}
$$

in which $(\bar{x}, \bar{y}),(\bar{u}, \bar{v})$ and $\bar{p}$ are the coordinates, velocity components and pressure in the wave frame.

Expression of an extra stress tensor for Sisko fluid is (Nadeem and Akbar 2010)

$\overline{\mathbf{S}}=\left[a+b(\sqrt{\bar{\Pi}})^{n-1}\right] \bar{A}_{1}$,

$\bar{A}_{1}=\overline{\mathbf{L}}+\overline{\mathbf{L}}^{\mathbf{T}}, \overline{\mathbf{L}}=\operatorname{grad} \overline{\mathbf{V}}, \bar{\Pi}=\frac{1}{2} \operatorname{tr}\left(\bar{A}_{1}^{2}\right)$,

in which $\overline{\mathbf{S}}$ is the extra stress tensor, $a$ and $b$ are material constant of Sisko fluid model.

Velocity stream function relation and non-dimensional quantities are $u=\frac{\partial \Psi}{\partial y}, v=-\delta \frac{\partial \Psi}{\partial x}$,

$$
\begin{aligned}
x= & \frac{2 \pi \bar{x}}{\lambda}, y=\frac{\bar{y}}{d_{1}}, u=\frac{\bar{u}}{c_{1}}, v=\frac{\bar{v}}{c_{1}}, t=\frac{2 \pi \bar{t}}{\lambda}, \delta=\frac{2 \pi d_{1}}{\lambda}, \\
& d=\frac{d_{2}}{d_{1}}, P=\frac{2 \pi d_{1}^{2} P}{\mu c_{1} \lambda}, \\
h_{1}= & \frac{\bar{h}_{1}}{d_{1}}, h_{2}=\frac{\bar{h}_{2}}{d_{2}}, \operatorname{Re}=\frac{\rho c_{1} d_{1}}{\mu}, a=\frac{a_{1}}{d_{1}}, b=\frac{a_{2}}{d_{1}}, \\
& d=\frac{d_{2}}{d_{1}}, S=\frac{\bar{S} d_{1}}{\mu c_{1}}, \\
\theta= & \frac{\bar{T}-\bar{T}_{0}}{\bar{T}_{1}-\bar{T}_{0}}, \sigma=\frac{\bar{C}-\bar{C}_{0}}{\bar{C}_{1}-\bar{C}_{0}}, \alpha=\frac{k}{(\rho c)_{\mathrm{f}}}, \\
& N_{\mathrm{b}}=\frac{(\rho c)_{\mathrm{p}} D_{\mathrm{B}}\left(\bar{C}_{1}-\bar{C}_{0}\right)}{(\rho c)_{\mathrm{f}} v}, b^{*}=\frac{b}{a\left(d_{1} / c\right)^{n-1}}, \\
P_{\mathrm{r}}= & \frac{v}{\alpha}, N_{\mathrm{t}}=\frac{(\rho c)_{\mathrm{p}} D_{\overline{\mathrm{T}}}\left(\bar{T}_{1}-\bar{T}_{0}\right)^{2}}{\bar{T}_{0}(\rho c)_{\mathrm{f}} v}, G_{\mathrm{r}}=\frac{g \alpha d_{1}^{2}\left(\bar{T}_{1}-\bar{T}_{0}\right)}{v c_{1}}, \\
B_{\mathrm{r}}= & \frac{g \alpha d_{1}^{2}\left(\bar{C}_{1}-\bar{C}_{0}\right)}{v c_{1}} .
\end{aligned}
$$

Using Eqs. (2) to (5) after using the long-wavelength and low Reynold's number approximation, we finally obtain the following system of equations:

$$
\begin{aligned}
& \frac{\partial^{2}}{\partial y^{2}}\left[\left(1+b^{*}\left|\frac{\partial^{2} \Psi}{\partial y^{2}}\right|^{n-1}\right) \frac{\partial^{2} \Psi}{\partial y^{2}}-M^{2} \Psi\right]+G_{\mathrm{r}} \frac{\partial \theta}{\partial y}+B_{r} \frac{\partial \sigma}{\partial y} \\
& \quad=0
\end{aligned}
$$

$\frac{\mathrm{d} P}{\mathrm{~d} x}=\frac{\partial}{\partial y}\left[\left(1+b^{*}\left|\frac{\partial^{2} \Psi}{\partial y^{2}}\right|^{n-1}\right) \frac{\partial^{2} \Psi}{\partial y^{2}}-M^{2} \Psi\right]+G_{\mathrm{r}} \theta+B_{\mathrm{r}} \sigma$,

$0=\frac{\partial^{2} \theta}{\partial y^{2}}+\operatorname{Pr} N_{\mathrm{b}} \frac{\partial \sigma}{\partial y} \frac{\partial \theta}{\partial y}+\operatorname{Pr} N_{\mathrm{t}}\left(\frac{\partial \theta}{\partial y}\right)^{2}$, 
$0=\left(\frac{\partial^{2} \sigma}{\partial y^{2}}\right)+\frac{N_{\mathrm{t}}}{N_{\mathrm{b}}}\left(\frac{\partial^{2} \theta}{\partial y^{2}}\right)$

Where $P_{\mathrm{r}}$ is the Prandtl number, $N_{\mathrm{b}}$ the Brownian motion parameter, $N_{\mathrm{t}}$ the thermophoresis parameter, $G_{\mathrm{r}}$ the local temperature Grashof number, $M$ the Hartmann number and $B_{\mathrm{r}}$ the local nanoparticle Grashof number.

Corresponding boundary conditions for asymmetric channel in nondimensional form take the following form:

$\psi=\frac{F}{2}, \frac{\partial \psi}{\partial y}=-1$, at $y=h_{1}=1+a \cos x$,
$\psi=-\frac{F}{2}, \frac{\partial \psi}{\partial y}=-1$, at $y=h_{2}=-d-b \cos (x+\phi)$

$\theta=0, \sigma=0, y=h_{1}=1+a \cos x$,

$\theta=1, \sigma=1, y=h_{2}=-d-b \cos (x+\phi)$

The time mean $Q$ (in the wave frame) are defined as

$Q=F+1+d$.

and the dimensionless pressure rise $\Delta P$ is defined as

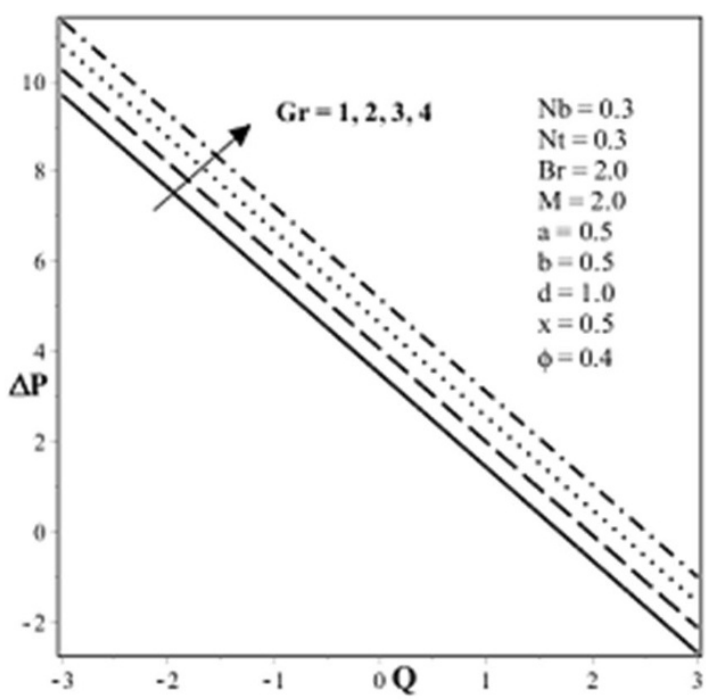

(b)

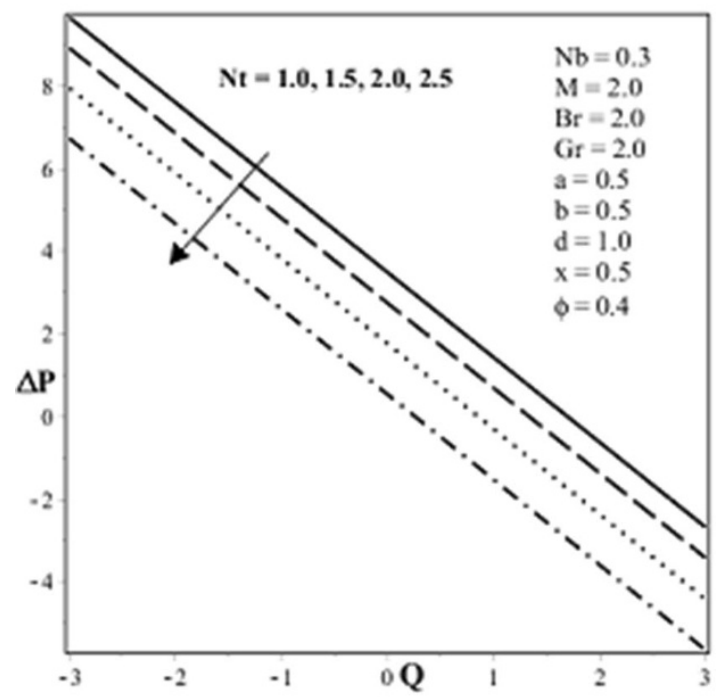

(c)

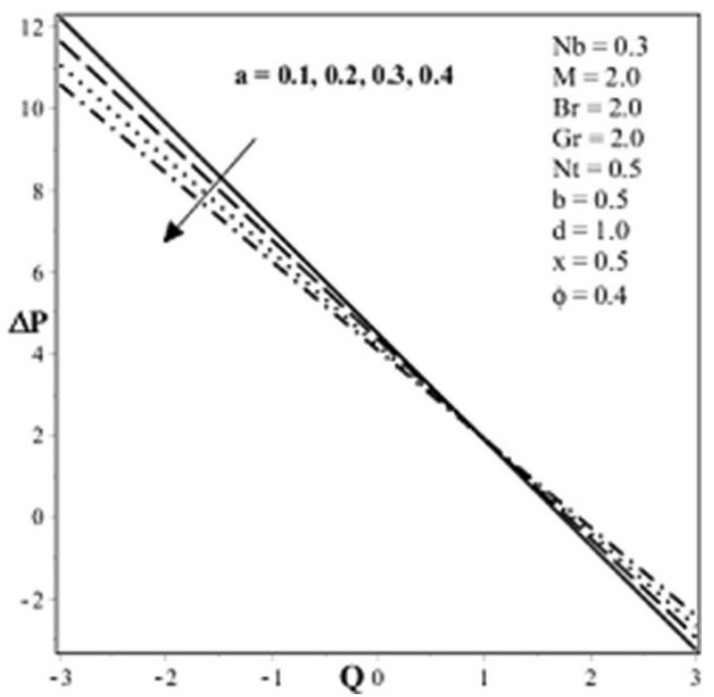

(d)

Fig. 1 a-d Pressure rise versus flow rate. $\mathbf{e}-\mathbf{h}$ Velocity profile 


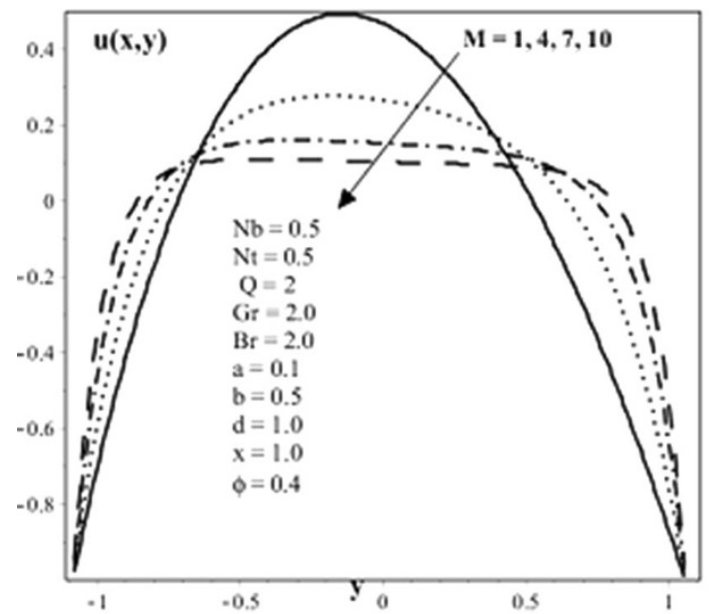

(e)

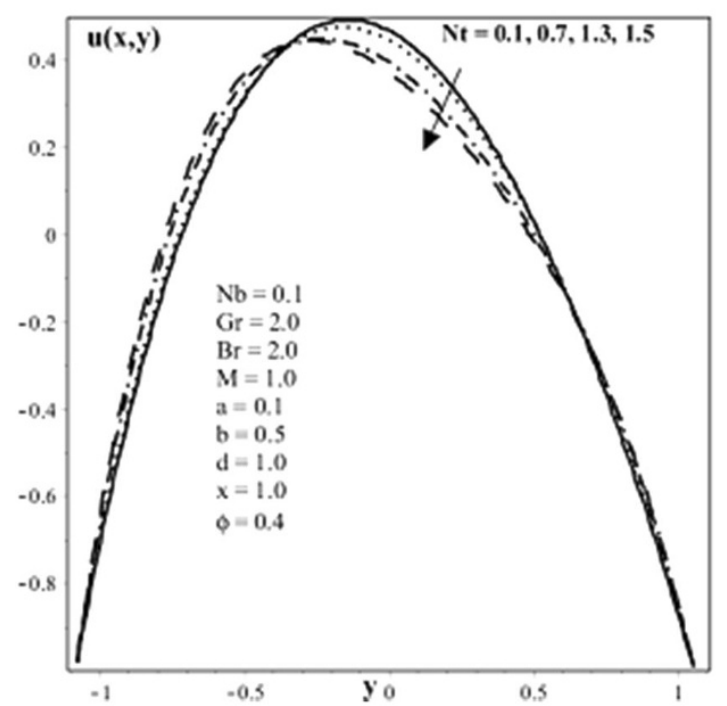

(g)

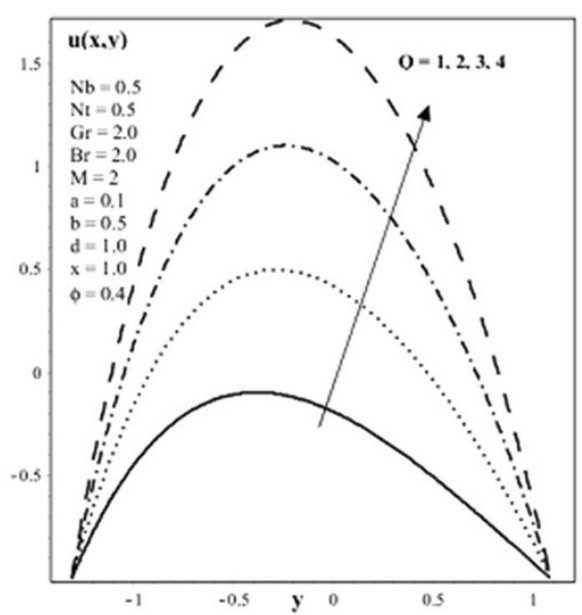

(f)

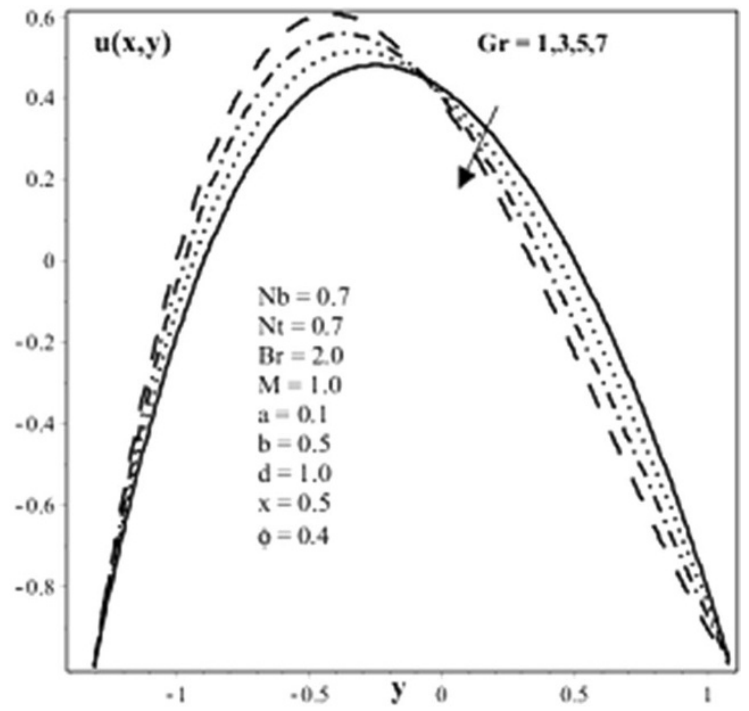

(h)

Fig. 1 continued

$$
\Delta P=\int_{0}^{1}\left(\frac{\mathrm{d} P}{\mathrm{~d} x}\right) \mathrm{d} x
$$

\section{Numerical Solution}

The system of coupled nonlinear coupled differential Eqs. (6-9) along with the boundary conditions $(11,13)$ is solved numerically using fourth-order Runge-Kutta-Fehlberg method with a shooting technique. The step size $\Delta y=0.001$ is used to obtain the numerical solution with $y_{\max }$ and an accuracy to the fifth decimal place is chosen as the criterion of convergence. The Runge-KuttaFehlberg method (or Fehlberg method) is an algorithm in numerical analysis for the numerical solution of ordinary differential equations. This gives us better convergent result as compared with some analytical techniques.

\section{Results and discussion}

We have examined the pressure rise, velocity, temperature, nanoparticle phenomena and streamlines for Sisko nano fluid model numerically and examined them through graphs. Three cases have been discussed with the different values of $n$. Here, we analyse the pressure rise per wavelength against volume flow rate which are portrayed in Figs. 1a-d, 2, 3a-d. It is observed that the 
pressure rise and volume flow rate show opposite behaviour. From Fig. $1 \mathrm{a}-\mathrm{d}$ it is also noticed that in pumping region $(\Delta P>0)$, the pressure rise increases with the increase in Hartmann number $M$ and temperature Grashof number $G_{\mathrm{r}}$. For the thermophoresis parameter $N_{\mathrm{t}}$ and amplitude ratio $a$, the pressure rise decreases when we increase the value of $a$ and $M$. Figure $1 \mathrm{a}-\mathrm{d}$ also shows that in the augmented pumping region for $(\Delta P<0)$, the pressure rise gives the opposite results for all the parameters as compared with peristaltic pumping region. Free pumping region holds when $(\Delta P=0)$. Figure $3 \mathrm{e}$ depicts that with an increase in Sisko fluid parameter $b^{*}$ pressure rise increases in the peristaltic pumping region while decreasing in the augmented pumping region. Variation of fluid parameters on velocity profile is shown in the Figs. $1 \mathrm{e}-\mathrm{h}, 2,3 \mathrm{f}-\mathrm{j}$. It depicts that the behaviour of velocity near the channel walls and at centre is not similar in view of the Hartmann number $M$. The velocity field increases with the increase in $M$ near the channel walls; however, it decreases in the centre of the channel. Velocity field increases with an increase in flow rate $Q$. It is observed that by increasing $N_{\mathrm{t}}$ and $G_{\mathrm{r}}$ velocity field increases in the region $y \in[-1.5,0]$ and decreases otherwise in the region $y \in[0.1,1.1]$. Velocity field decreases in the region $y \in[-1.5,0]$ and increases otherwise in the

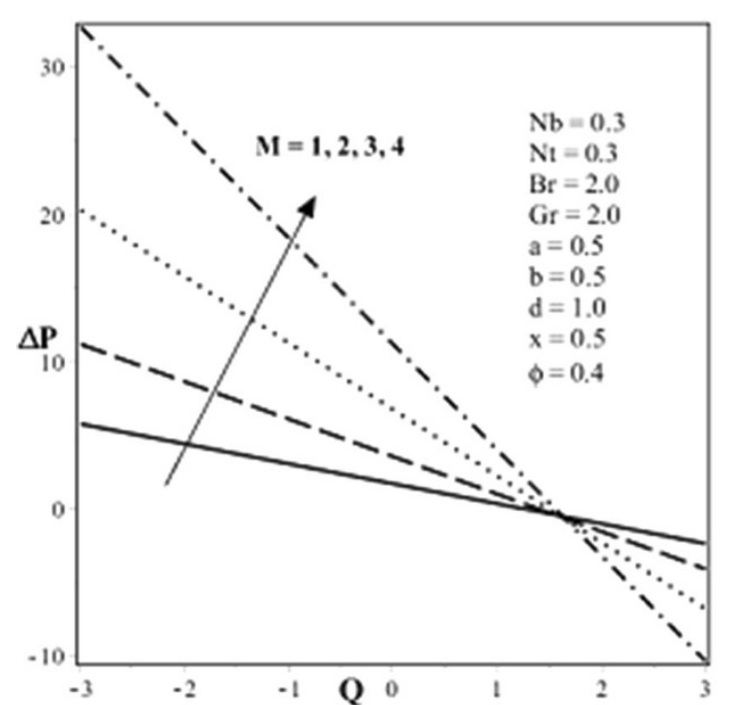

(a)

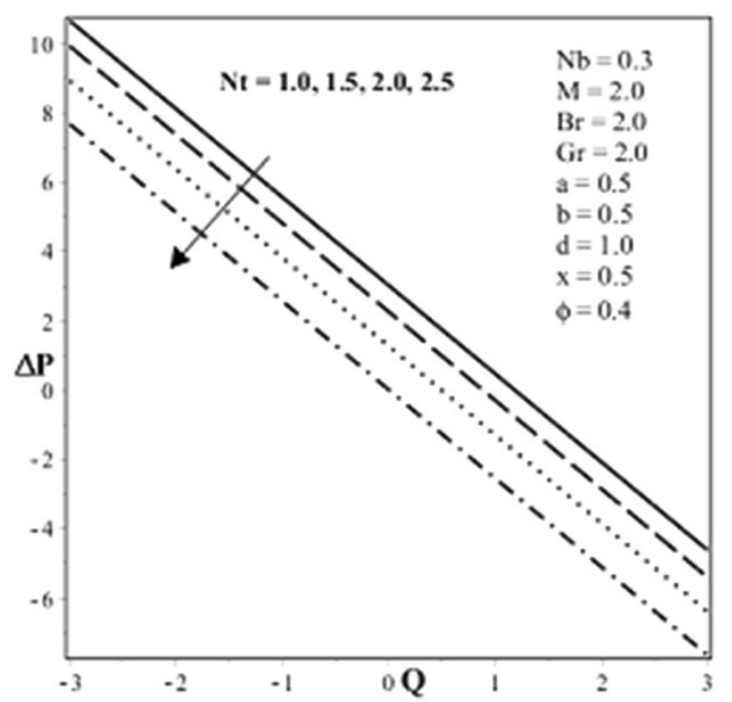

(c)

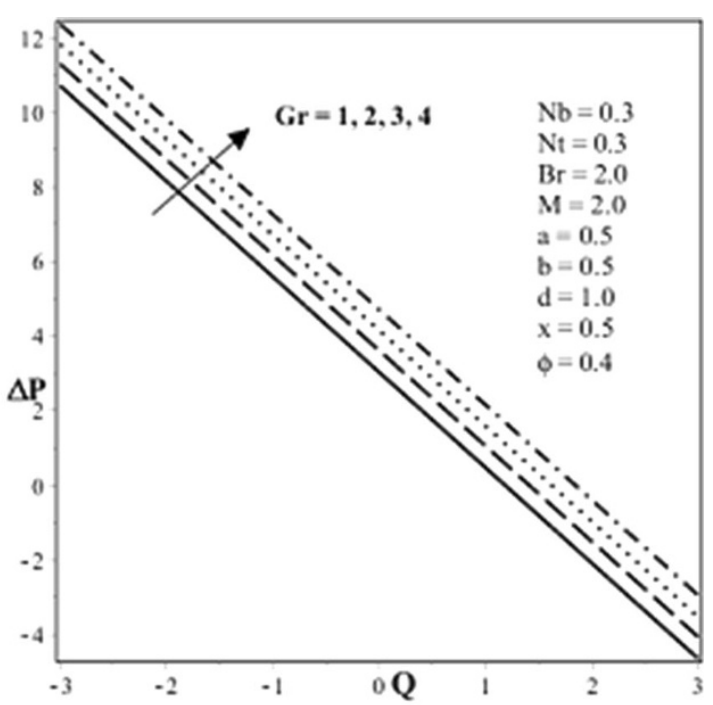

(b)

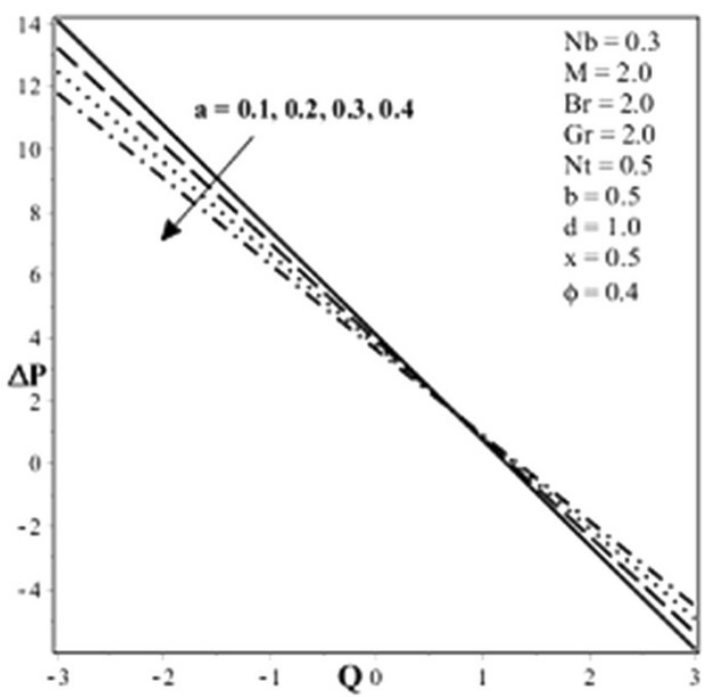

(d)

Fig. 2 a-d Pressure rise versus flow rate. $\mathbf{e}-\mathbf{h}$ Nano particle profile 


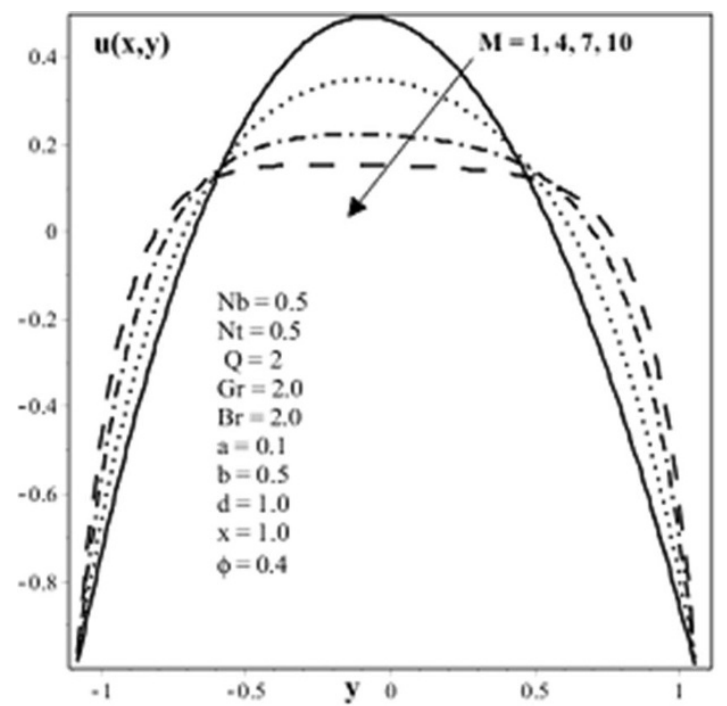

(e)

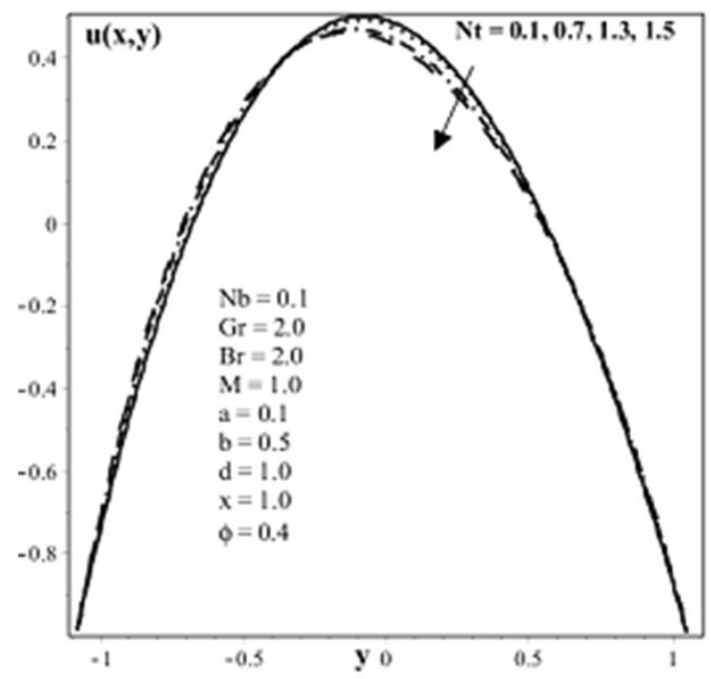

(g)

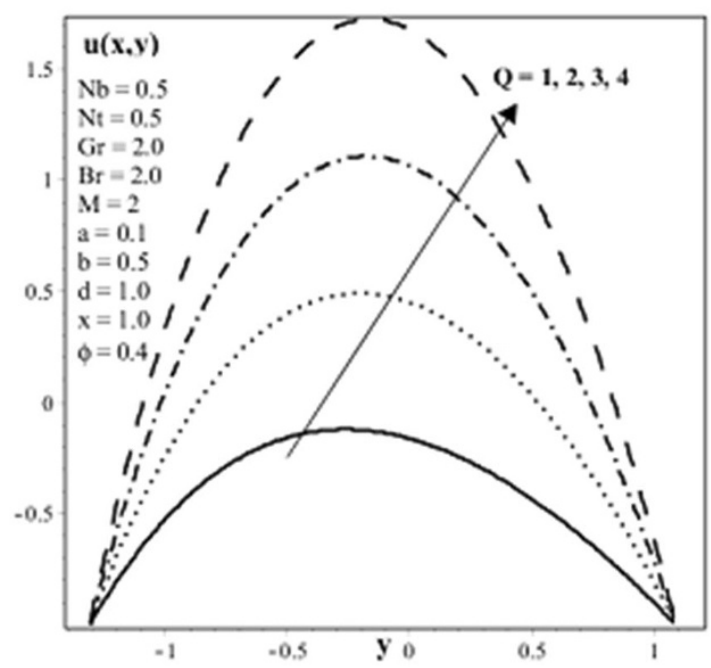

(f)

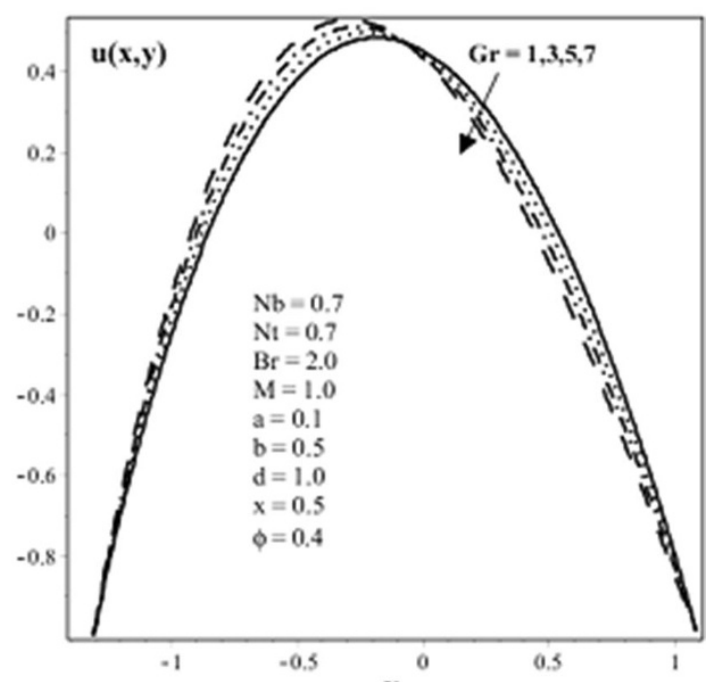

(h)

Fig. 2 continued

region $y \in[0.1,1.1]$ when the value of Sisko fluid parameter $b^{*}$ increases.

The trapping for different values of Sisko fluid parameter $b^{*}$ is shown in Fig. $4 \mathrm{a}, \mathrm{b}$. It is seen from Fig. $4 \mathrm{a}, \mathrm{b}$ that the size of the trapping bolus increases with an increase in $b^{*}$ while the number of bolus decreases.

The variation of temperature profile and Nano particle phenomena for different values of thermophoresis parameter $N_{\mathrm{t}}$ Prandtl parameter $P_{\mathrm{r}}$ and Brownian motion parameter $N_{\mathrm{b}}$ are plotted in the Figs 5 and 6 . Here the temperature profile increases when thermophoresis parameter $N_{\mathrm{t}}$ Prandtl parameter $P_{\mathrm{r}}$ and Brownian motion parameter $N_{\mathrm{b}}$ are increased, and nanoparticle phenomena decreases when there is an increase in the values of thermophoresis parameter $N_{\mathrm{t}}$, Prandtl parameter $P_{\mathrm{r}}$ and increases with an increase in Brownian motion parameter $N_{\mathrm{b}}$.

\section{Concluding remarks}

The present study discussed the peristaltic flow of a Nano Sisko fluid in an asymmetric channel. The key points of the performed analysis are as follows:

1. The pressure rise increases with the increase in Hartmann number $M$ and temperature Grashof 
number $G_{\mathrm{r}}$. For the thermophoresis parameter $N_{\mathrm{t}}$ and amplitude ratio $a$, the pressure rise decreases when we increased the value of $a$ and $M$.

2. The pressure rise gives the opposite results for all the parameters as compared with peristaltic pumping region.

3. Free pumping region holds when $(\Delta P=0)$.

4. With an increase in Sisko fluid parameter $b^{*}$ pressure rise increases in the peristaltic pumping region while decreasing in the augmented pumping region.

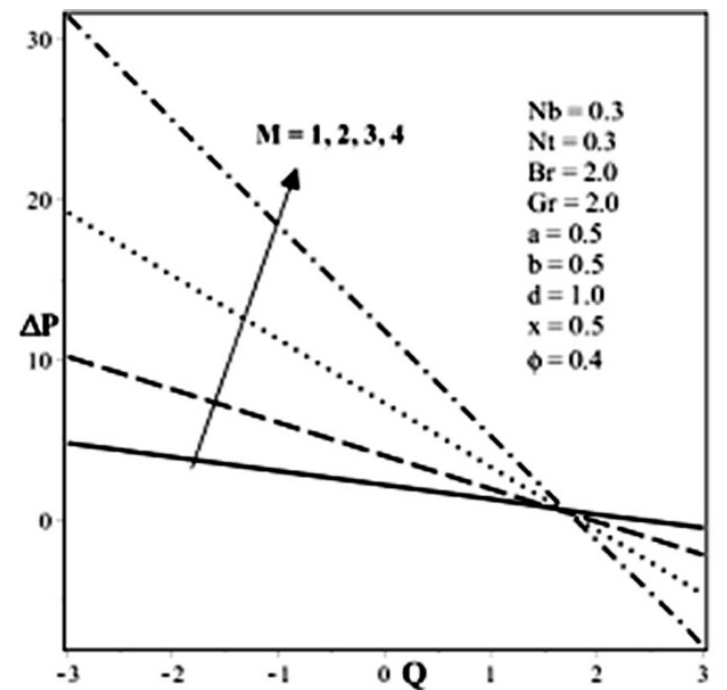

(a)

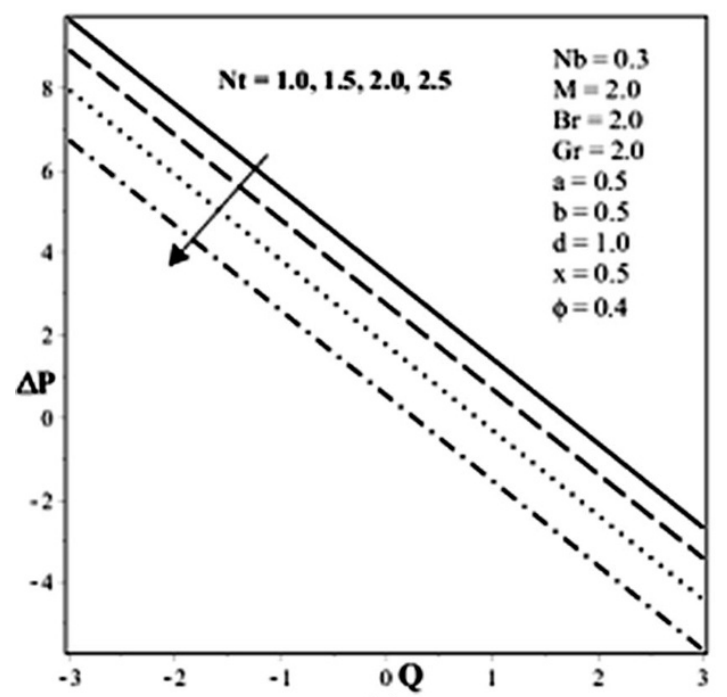

(c)
5. The behaviour of velocity near the channel walls and at center is not similar in view of the Hartmann number $M$.

6. The velocity field increases with the increase in $M$ near the channel walls; however, it decreases in the centre of the channel.

7. Velocity field increases with an increase in flow rate $Q$.

8. Velocity field decreases in the region $y \in[-1.5,0]$ and increases otherwise in the region $y \in[0.1,1.1]$ when the value of Sisko fluid parameter $b^{*}$ increases.

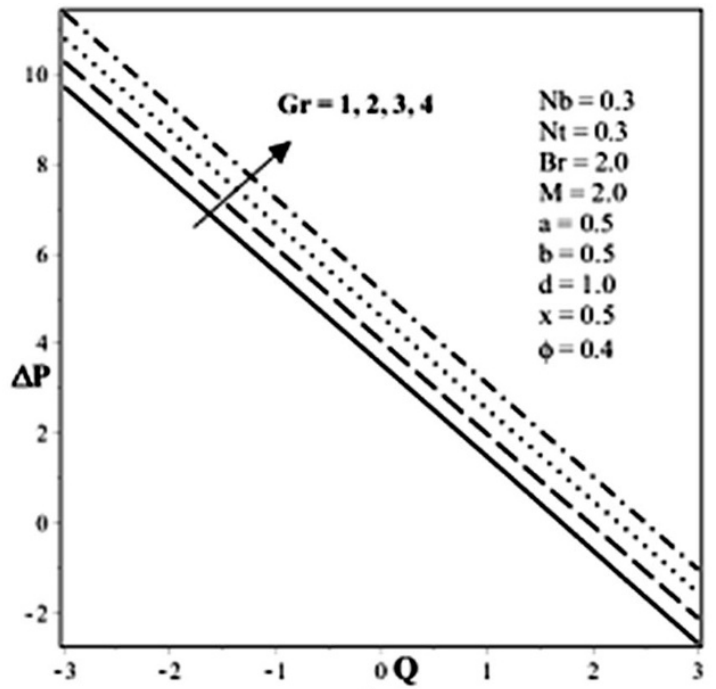

(b)

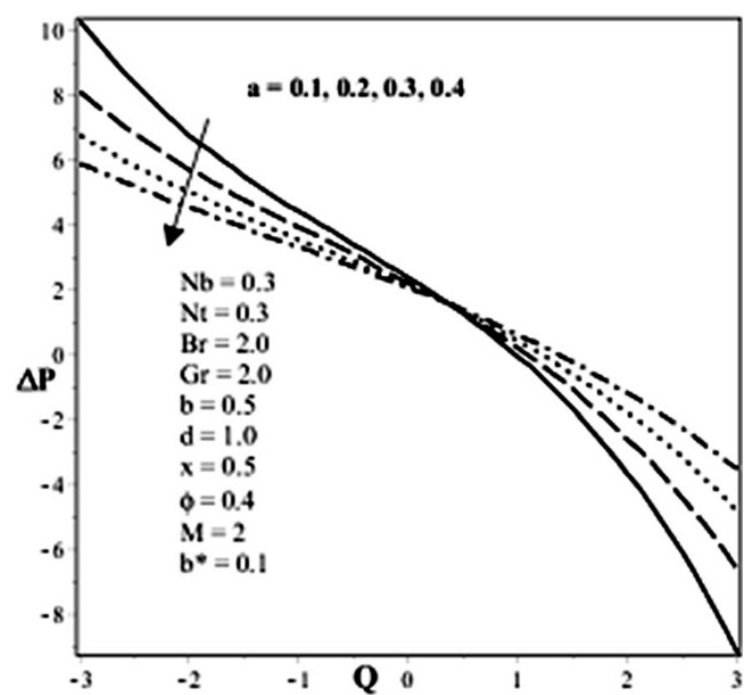

(d)

Fig. 3 a-e Pressure rise versus flow rate. $\mathbf{f}-\mathbf{j}$ Pressure rise versus flow rate 


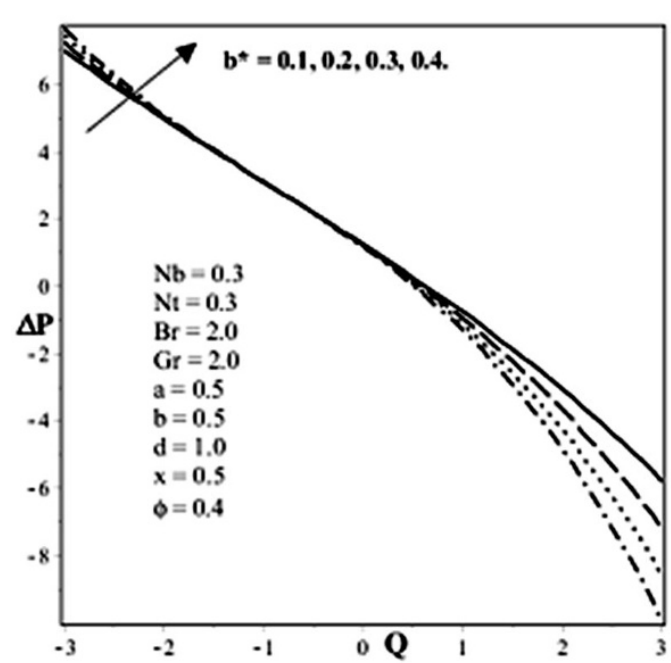

(e)

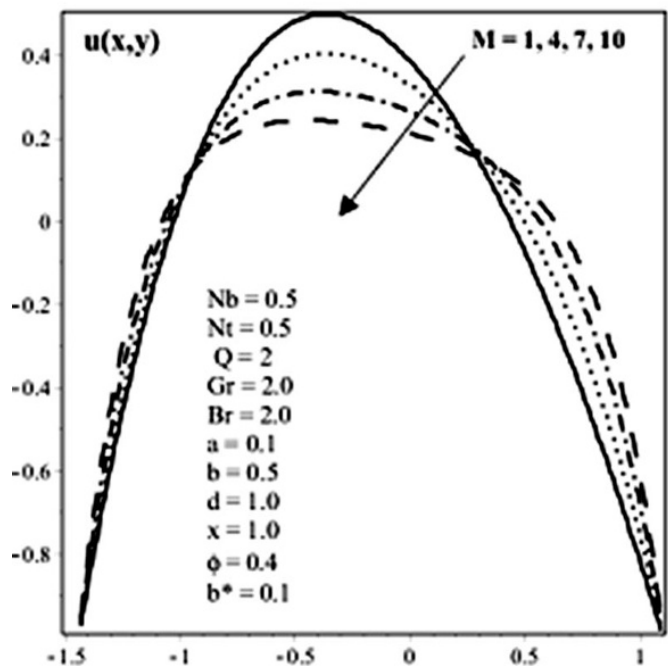

(g)

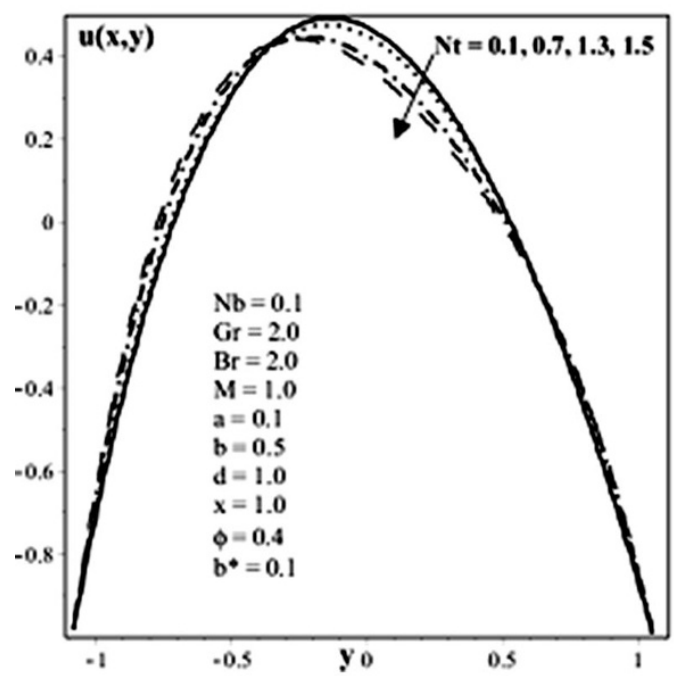

(i)

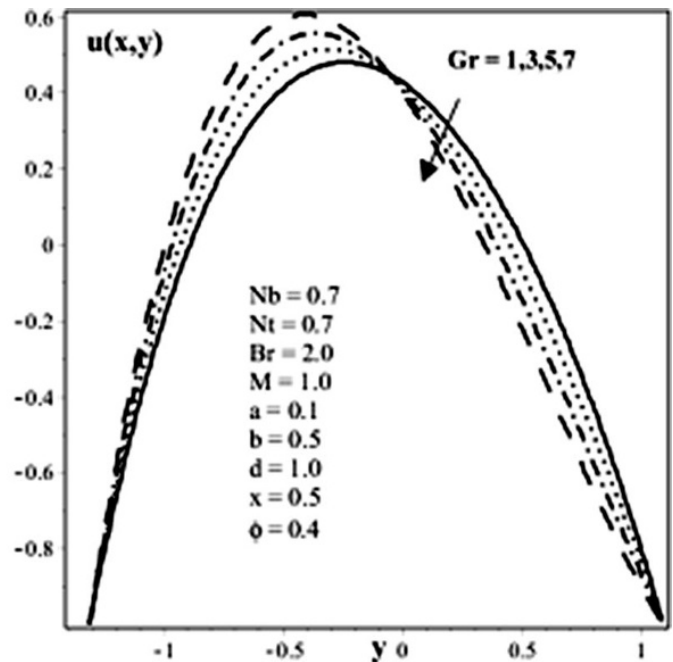

(f)

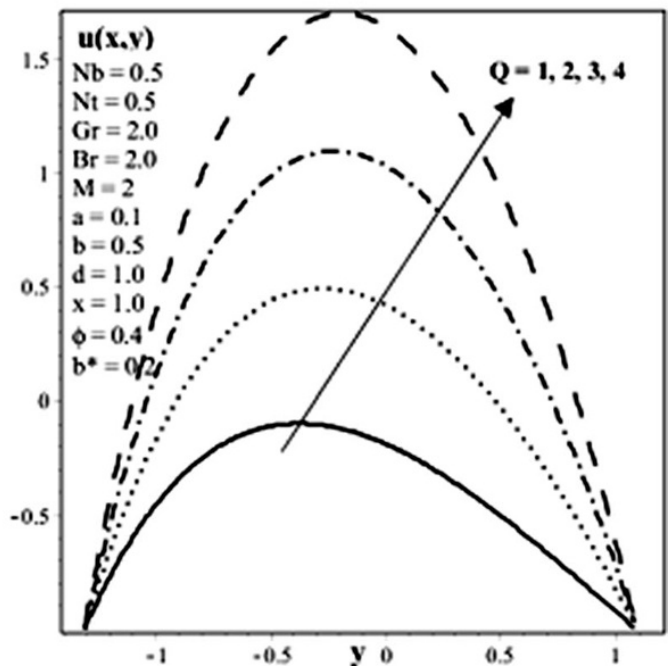

(h)

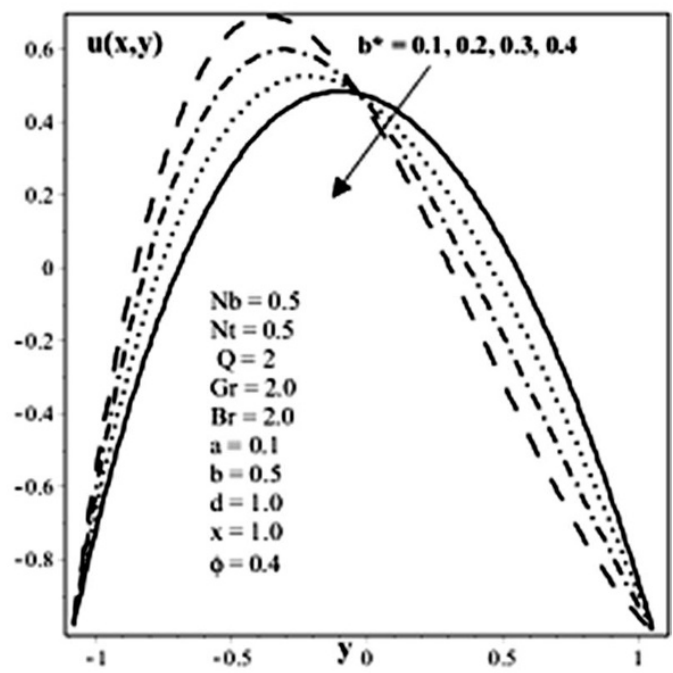

(j)

Fig. 3 continued 


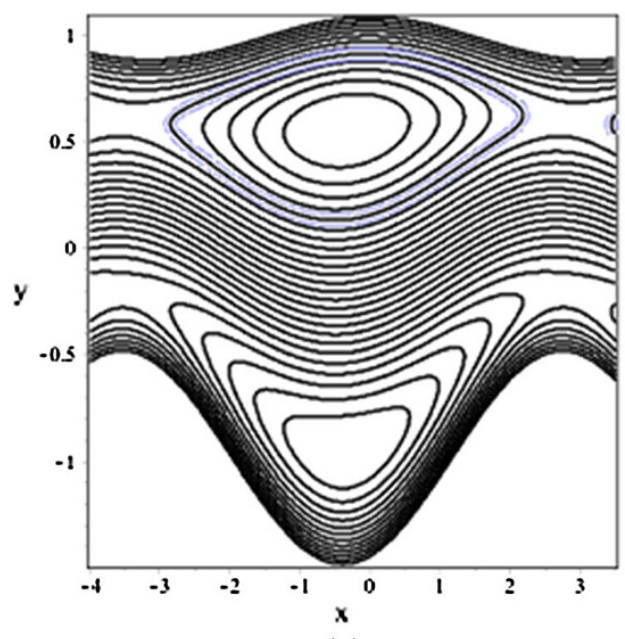

(a)

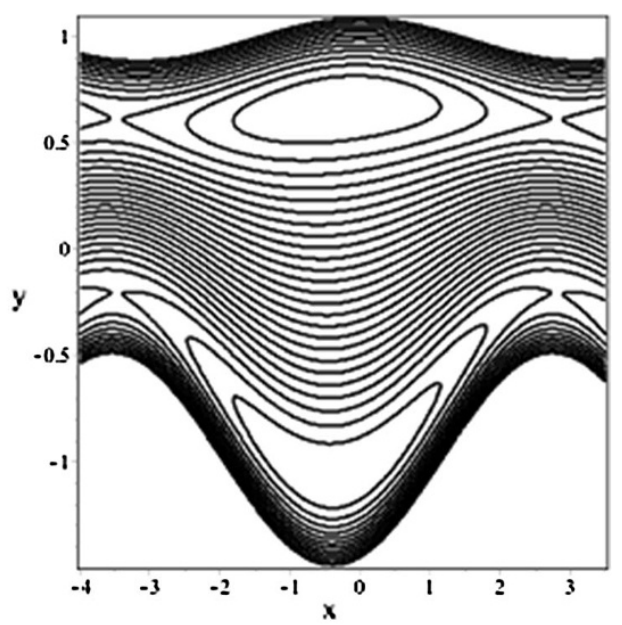

(b)

Fig. 4 Streamlines $(\mathbf{a}, \mathbf{b}) b^{*}=0.2,0.4$. The other parameters are $a=0.5, b=0.5 d=1, \phi=0.4, G_{\mathrm{r}}=2, B_{\mathrm{r}}=2, N_{\mathrm{b}}=0.5, N_{\mathrm{t}}=0.5$, $M=1, P_{\mathrm{r}}=1$

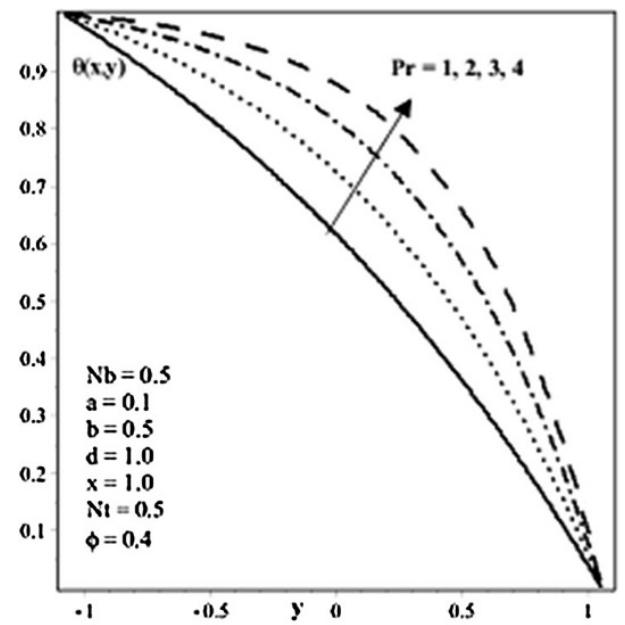

(a)

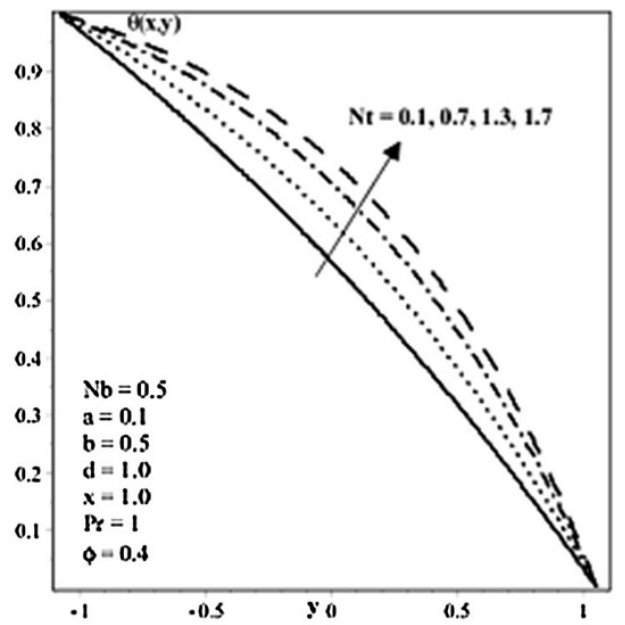

(b)

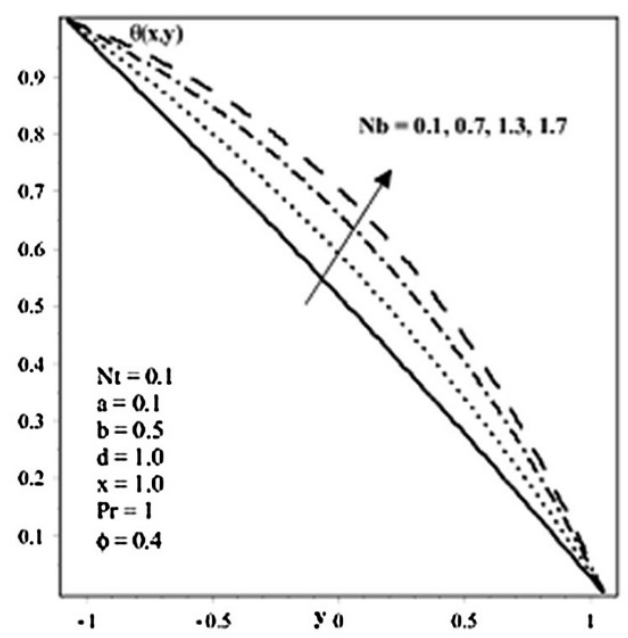

(c)

Fig. 5 a-c Temperature profile 


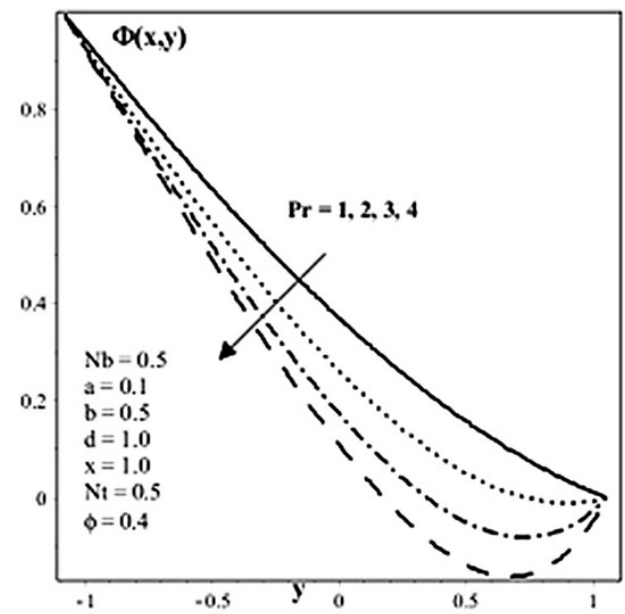

(a)

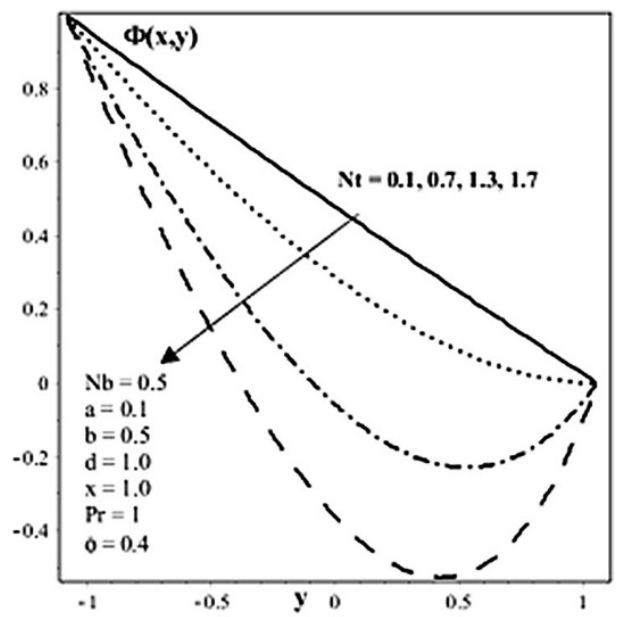

(b)

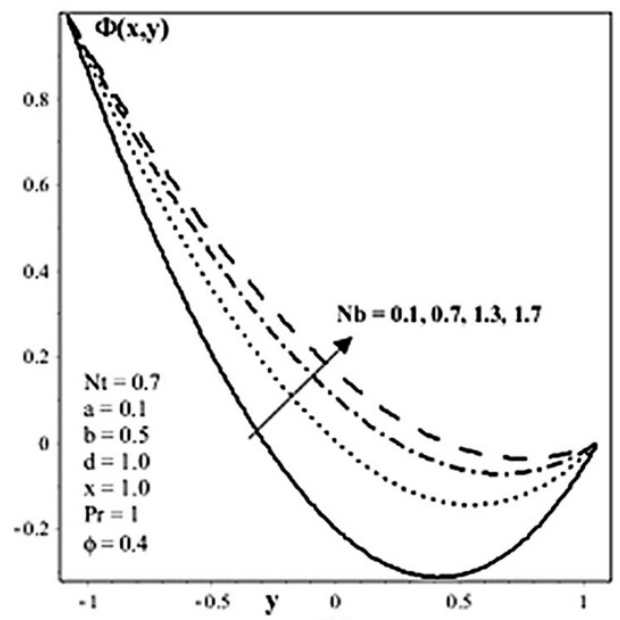

(c)

Fig. 6 a-c Nano particle profile

9. The temperature profile increases when thermophoresis parameter $N_{\mathrm{t}}$, Prandtl parameter $P_{\mathrm{r}}$ and Brownian motion parameter $N_{\mathrm{b}}$ are increased.

10. Nanoparticle phenomena decreases when there is an increase in the values of thermophoresis parameter $N_{\mathrm{t}}$, Prandtl parameter $P_{\mathrm{r}}$ and increases with an increase in Brownian motion parameter $N_{\mathrm{b}}$

11. The size of the trapping bolus increases with an increase in $b^{*}$ while number of bolus decreases.

Open Access This article is distributed under the terms of the Creative Commons Attribution License which permits any use, distribution, and reproduction in any medium, provided the original author(s) and the source are credited.

\section{References}

Akbar NS, Nadeem S (2011) Endoscopic effects on the peristaltic flow of a nanofluid. Commun Theor Phys 56:761-768
Akbar NS, Nadeem S (2012a) Thermal and velocity slip effects on the peristaltic flow of a six constant Jeffrey's fluid model. Int J Heat Mass Transf 55:3964-3970

Akbar NS, Nadeem S (2012b) Peristaltic flow of a Phan-Thien-Tanner nanofluid in a diverging tube. Heat Transf Res 41:10-22

Akbar NS, Nadeem S, Lee C (2012a) Peristaltic flow of a Prandtl fluid model in an asymmetric channel. Int J Phys Sci 7:687-695

Akbar NS, Nadeem S, Hayat T, Hendi AA (2012b) Peristaltic flow of a nano fluid with slip effects. Meccanica 47:1283-1294

Akbar NS, Nadeem S, Hayat T, Hendi AA (2012c) Peristaltic flow of a nanofluid in a non-uniform tube. Heat Mass Transf 48:451-459

Akram S, Nadeem S, Ghafoor A, Lee C (2012) Consequences of nanofluid on Peristaltic flow in an asymmetric channel. Int $\mathbf{J}$ Basic Appl Sci IJBAS-IJENS 12(5):75-96

Aziz A, Khan WA (2012) Natural convective boundary layer flow of a nanofluid past a convectively heated vertical plate. Int $\mathbf{J}$ Thermal Sci 52:83-90

Choi SUS (1995) Enhancing thermal conductivity of fluids with nanoparticles. In: Siginer DA, Wang HP (eds) Developments and applications of non-Newtonian flows, vol 66. ASME, New York, pp 99-105

El-Shehawey EF, Husseny SZA (2002) Peristaltic transport of a magneto-fluid with porous boundaries. Appl Math Comput 129:421-440 
Elshehawey FE, Sobh AMF, Afify NAA (2000) Peristaltic motion of a generalized Newtonian fluid under the effect of transverse magnetic field. Comptes Rendus de l'Academie Bulgare des Sciencesi 53:33-38

Hamad MAA, Ferdows M (2012) Similarity solution of boundary layer stagnation-point flow towards a heated porous stretching sheet saturated with a nanofluid with heat absorption/generation and suction/blowing: a lie group analysis. Commun Nonlinear Sci Numer Simulat 17:132-140

Jaffrin MY, Shapiro AH (1971) Peristaltic pumping. Annu Rev Fluid Mech 37:13-37

Kothandapani M, Srinivas S (2008) Non-linear peristaltic transport of a Newtonian fluid in an inclined asymmetric channel through a porous medium. Phys Lett A 372:1265-1276

Latham TW (1966) Fluid motion in a peristaltic pump, MS. Thesis, Massachusetts Institute of Technology, Cambridge
Marga SEB, Palm SJ, Nguyen CT, Roy G, Galanis N (2005) Heat transfer enhancement by using nanofluids in forced convection flows. Int J Heat Fluid Flow 26:530-546

Mekheimer KhS (2008) Effect of the induced magnetic field on peristaltic flow of a couple stress fluid. Phys Lett A 372:4271-4278

Nadeem S, Akbar NS (2010) Peristaltic flow of Sisko fluid in a uniform inclined tube. Acta Mech Sin 26:675-683

Sadik K, Pramuanjaroenkij A (2009) Review of convective heat transfer enhancement with nanofluids. Int $\mathrm{J}$ Heat Mass Transf 52:3187-3196

Saleh H, Roslan R, Hashima I (2011) Natural convection heat transfer in a nanofluid-filled trapezoidal enclosure. Int $\mathrm{J}$ Heat Mass Transf 54:194-201

Srinivas S, Kothandapania M (2009) The influence of heat and mass transfer on MHD peristaltic flow through a porous space with compliant walls. Appl Math Comput 213:197-208 